\title{
OPTICAL CORRELATION OF FUNCTIONS DISTORTED AS $f\left(x^{\prime}\right)=f\left(x^{a}\right)$
}

\author{
Demetri PSALTIS and David CASASENT \\ Carnegie-Mellon University, Department of Electrical Engineering, Pittsburgh, PA 15213, USA
}

Received 10 February 1977

The use of coordinate transformation preprocessing is used to produce a space-variant optical correlator invariant to functions distorted as $f\left(x^{\prime}\right)=f\left(x^{a}\right)$. Such distortions arise in non-linear scanning devices, imaging from curved surfaces, aberrations in an inaging system, and in the Doppler shift of target with non-uniform motion.

\section{Introduction}

The use of a coordinate transformation preprocessor and a space invariant optical system has been found most useful in producing space variant optical processors [1]. The use of such techniques in synthesizing optical pattern recognition systems invariant to input distortions such as scale [2] and rotation [3], and their use in signal processing [4-5] have recently been reported. We now report on the use of space variant optical pattern recognition by coordinate transformation in the correlation of functions in which the reference $f(x)$ and input $f\left(x^{\prime}\right)=f^{\prime}(x)$ functions are distorted versions of one another, with the distortion described by

$f^{\prime}(x)=f\left(x^{\prime}\right)=f\left(x^{a}\right)$.

Such input coordinate distortions are representative of those that would be produced by non-linear scanning devices, aberrations in an optical imaging system [6], imaging from curved surfaces [7], and are descriptive of the Doppler shifts due to non-uniform target or platform motion [8].

\section{Theoretical considerations}

The first step in the realization of a distortion invariant optical correlator is to apply a coordinate transformation $h^{-1}(x)=\xi$ to the input and reference functions to produce new functions $f_{1}(\xi)$ and $f_{1}^{\prime}(\xi)$ with the coordinates transformed. These are then used as the input and reference functions to a conventional space invariant optical correlator. If the coordinate transformation is properly chosen so that the distortion in the input $x$ space is converted to a shift by $\xi_{0}$ in the coordinate transformed $\xi$ space, i.e.

$h^{-1}(x)-h^{-1}\left(x^{\prime}\right)=\xi_{0}$,

then the resultant system will be space variant and invariant to the desired input distortion. The signal-tonoise ratio of the output correlation peak will be the same for the cross-correlation of the original function and a distorted version of it as it is for the autocorrelation of the original function. Furthermore, the location of the output correlation peak will be proportional to the distortion parameter $a$, and hence $a$ can be determined. The system requires no a-priori knowledge of the parameter $a$, only that the distortion is of the general form

$x^{\prime}=g(x, a)=x^{a}$.

One-dimensional functions are considered throughout for simplicity only.

In terms of the general distortion $x^{\prime}=g(x, a)$, we have shown that the coordinate transformation $h^{-1}(x)=\xi$ must satisfy

$h^{-1}(x)=-\frac{\mathrm{d} \xi_{0}}{\mathrm{~d} a} \int_{-\infty}^{x} \frac{\partial g(x, a) / \partial x}{\partial g(x, a) / \partial a} \mathrm{~d} x$,

where $\xi_{0}$ is a function of $a$ only. For the distortion 
under consideration described by eq. (3), we find

$h^{-1}(x)=-\frac{\mathrm{d} \xi_{0}}{\mathrm{~d} a} \int_{-\infty}^{x} \frac{a x^{a-1}}{x^{a} \ln x} \mathrm{~d} x=-\frac{\mathrm{d} \xi_{0}}{\mathrm{~d} a} \int_{-\infty}^{x} \frac{a \mathrm{~d} x}{x \ln x}$

Setting $\xi_{0}=-\ln a$, we find the required coordinate transformation to be

$\xi=h^{-1}(x)=\ln (\ln x)$.

This transformation has a one-to-one correspondence and its inverse exists,

$x=h(\xi)=\exp (\exp \xi)$.

As specified in eq. (2), this transformation should convert an exponentiation of the input $x$ coordinates into a shift by $\xi_{0}$ in $\xi$ space. Substitution of eq. (7) into eq. (2) yields

$$
\begin{aligned}
x^{a} & =[h(\xi)]^{a}=[\exp (\exp \xi)]^{a} \\
& =\exp (a \exp \xi)=\exp [\exp (\xi+\ln a)] \\
& =h(\xi+\ln a)=h\left(\xi-\xi_{0}\right),
\end{aligned}
$$

which satisfied eq. (2) with $\xi_{0}=-\ln a$ in agreement with the analysis of eq. (4).

\section{Implementation}

The distortion invariant system described above can be realized: (a) by a system with an input coordinate transformation described by eq. (6) followed by a space invariant optical correlator, or (b) by using the magnitude of the Mellin transforms of the functions as inputs to a scale invariant correlator. We previously showed [2] that a Mellin transform correlator was scale invariant and that the optical Mellin transform $M(u)$ of $f(x)$ could be realized by forming the Fourier transform of $f\left(\mathrm{e}^{\xi_{1}}\right)$, where $\xi_{1}=\ln x$.

The difference between these two implementations may appear minor, but a brief discussion will provide considerable insight into these correlators and space variant processing by coordinate transformation.

The Mellin transform correlation method (b) involves a single logarithmic scaling of the coordinates of the input function (rather than two successive logarithmic coordinate transformations) followed by formation of the Fourier transform of these coordinate transformed functions. These two Mellin transforms are then used as inputs to the conventional optical scale invariant correlator, rather than the double logarithmically coordinate scaled functions. Thus, this second method (b) is equivalent to performing two successive Mellin transforms.

Since coordinate transformations can only be performed on real functions, the magnitude of the first Mellin transform must be formed in method (b) and used as the input to the scale invariant correlator. As a result, the phase information present in the Mellin transform of the input function is lost in implementation method (b). Conversely, in method (a), the $0 \geqslant x>1$ region of the full input function extending from $0 \geqslant x>\infty$ will map onto the negative region of the logarithmically scaled function. Data present in this $0 \geqslant x>1$ region of the input function will be lost since one cannot form the logarithm of a negative number. The application and specific input data used will determine which implementation method is best.

\section{Experimental confirmation}

To demonstrate the feasibility of coordinate transformation preprocessing for invariance to distortions described by eq. (1), a simple input grating (fig. 1a) was used as the reference function $f(x)$. The input function $f^{\prime}(x)$ subjected to the distortion described by eq. (1) with $a=1.2$ is shown in fig. $1 \mathrm{~b}$. The input $f_{1}(\xi)$ after application of the coordinate transformation described by eq. (5) is shown in fig. $1 \mathrm{c}$. These coordinate transformations can be realized on real-time devices by analog circuit modules in the deflection system and by computer generated hologram masks as previously described. The first logarithmic conversion converts the coordinate exponentiation into a scaling. The second logarithmic conversion then converts the scaling into a shift.

These coordinate transformed functions $f_{1}(\xi)$ and $f_{1}^{\prime}(\xi)$ are then used as the reference and input functions to a conventional Van der Lugt frequency plane correlator [9]. A matched spatial filter of $f_{1}(\xi)$ was formed and properly repositioned in the filter plane. With $f_{1}^{\prime}(\xi)$ placed in the input plane, the output correlation plane contains the desired correlation $f \circledast f^{\prime}$ shown in fig. $1 \mathrm{~d}$. The cross-sectional scan of the autocorrelation $f \circledast f$ is shown in fig. 1e and the cross- 


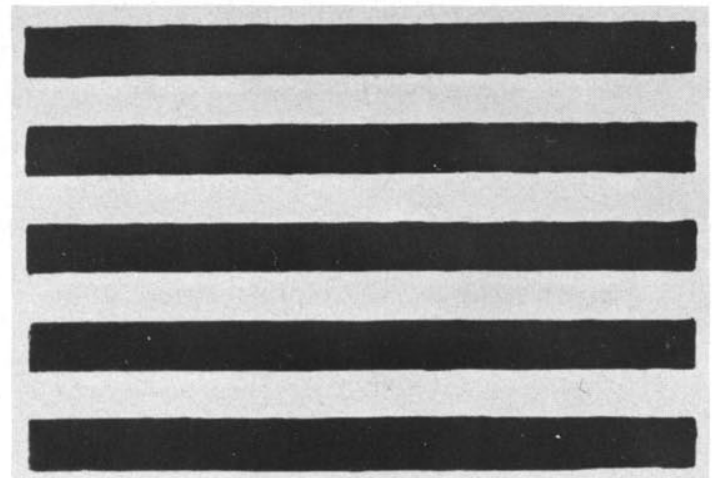

Fig. 1a.

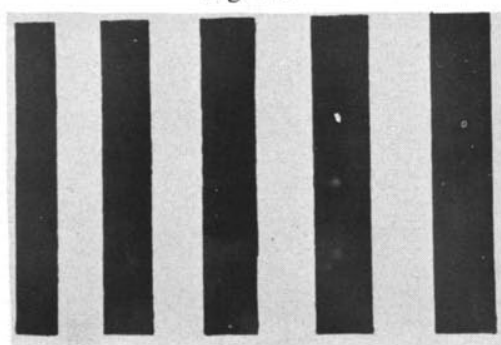

Fig. 1b.

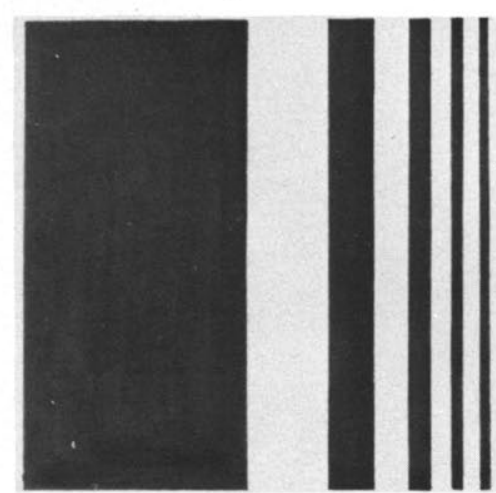

Fig. 1c.

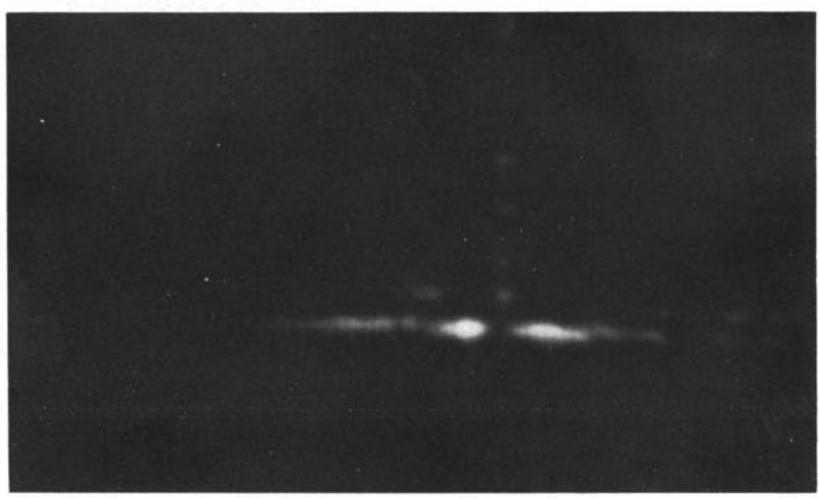

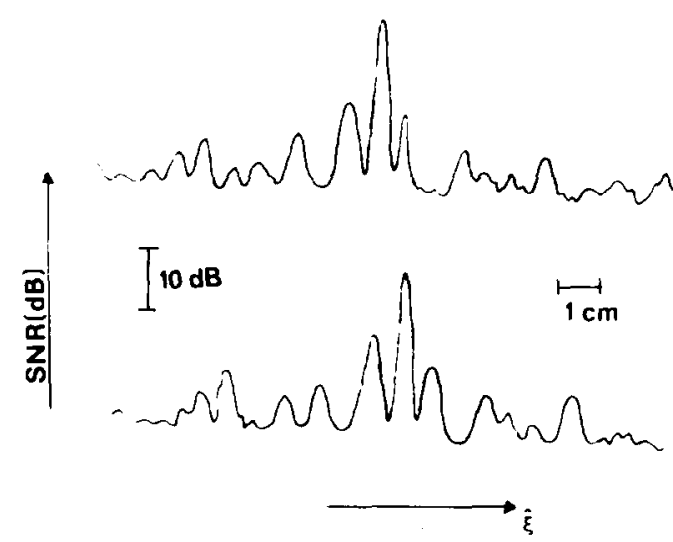

Fig. 1e. and Fig. 1f.

Fig. 1. Distortion invariant optical correlation. (a) reference function, (b) distorted input function with $x^{\prime}=x^{1.2}$, (c) coordinate transformed version of input function, (d) spacevariant, output optical correlation of input and reference functions, (e) cross-section of autocorrelation of reference function, $(f)$ cross-section of cross-correlation of input and reference functions.

sectional plot of the cross-correlation $f \circledast f^{\prime}$ in fig. 1f. As predicted, there is no loss in signal-to-noise ratio verifying the distortion invariance of this space variant correlator. The displacement of the cross-correlation peak from the reference location of the auto-correlation peak can also be shown to be proportional to the logarithm of the distortion parameter $\boldsymbol{a}$.

\section{Summary}

Another new application of space variant optical pattern recognition has been presented. The formulation of the requisite input coordinate transformation has been derived from general formuli for the case of a generalized input distortion. Two implementations of the distortion invariant correlator were discussed and tradeoffs advanced between the loss of phase information and the loss of the portion of the input data near $x=0$.

\section{Acknowledgement}

The support of AFOSR Air Systems Command USAF on Grant AFOSR-75-2851 and BMDATC 
Huntsville on Contract DASG 60-77-C-0034 are gratefully acknowledged.

\section{References}

[1] D. Casasent and D. Psaltis, Prov. IEEF, 65 (1977).

[2] D. Casasent and D. Psaltis, Opt. Commun. 17 (1976) 59.

[3] D. Casasent and D. Psaltis, Appl. Opt. 15 (1976) 1795.
[4] D. Casasent and D. Psaltis, Appl. Opt. 15 (1976) 2015.

[5] D. Casasent and M. Kraus, Opt. Commun. 19 (1976) 212.

[6] W. Welfors, Aberrations of the symmetrical optical system, (Academic Press, London, 1974).

[7] W.H. Lee and O. Bryngdahl, Opt. Commun. 12 (1974) 382.

[8] E. Kelly and R. Wisher, IEEE Trans. Mil. Electr., MIL-9 (1965) 56.

[9] A. Van der Lught, IEEE Trans. Info. Theory, IT-10 (1965) 139. 